\title{
Trautmann-focused mastoidectomy for a simple, safe presigmoid approach: technical note
}

\author{
Kuniaki Tanahashi, MD, PhD, Kenji Uda, MD, PhD, Yoshio Araki, MD, PhD, \\ Kazuhito Takeuchi, MD, PhD, Jungsu Choo, MD, Lushun Chalise, MD, PhD, \\ Kazuya Motomura, MD, PhD, Fumiharu Ohka, MD, PhD, Toshihiko Wakabayashi, MD, PhD, and \\ Atsushi Natsume, MD, PhD
}

Department of Neurosurgery, Nagoya University Graduate School of Medicine, Nagoya, Japan

\begin{abstract}
The presigmoid approach (PSA) is selected to obtain more lateral access to cerebellopontine angle tumors, brainstem cavernous malformations, or vertebrobasilar artery aneurysms than the standard retrosigmoid approach. However, mastoidectomy for the PSA can be considered time-consuming and to carry a higher risk of complications due to the anatomical complexity of the region. The authors established a method of minimized mastoidectomy focused on exposing Trautmann's triangle as the corridor for the PSA while maximizing procedural simplicity and safety and maintaining a sufficient operative view. The authors present their method of minimized mastoidectomy in a cadaver dissection and operative cases, showing potential as a useful option for the PSA.
\end{abstract}

https://thejns.org/doi/abs/10.3171/2020.1.JNS193179

KEYWORDS presigmoid retrolabyrinthine approach; posterior petrosal approach; mastoidectomy; cerebellopontine angle tumor; brainstem cavernous malformation; vertebrobasilar artery aneurysm; surgical technique; skull base

$\mathrm{T}$ HE presigmoid approach (PSA), also called the retrolabyrinthine approach or posterior petrosal approach, is applied to large cerebellopontine angle (CPA) tumors compressing the brainstem, anterolateral pontine cavernous malformations, and vertebrobasilar artery aneurysms. This approach can be combined with another approach, including the anterior petrosal approach and retrosigmoid approach (RSA). In patients for whom the hearing should be maintained, the bony labyrinth is preserved..$^{1-4}$ The PSA provides a more lateral trajectory to these lesions than the standard RSA, with reductions in both operative distance and cerebellar retraction. ${ }^{5}$ However, mastoidectomy can be considered time-consuming and can harbor a higher risk of complications due to the anatomical complexity. We established a method of minimized mastoidectomy focused on exposing the presigmoid dura mater, Trautmann's triangle (TT), as the corridor for the PSA while maximizing the simplicity and safety of the procedure and maintaining a sufficient operative view. We present our method of minimized mastoidectomy in a cadaver dissection and operative cases, and suggest this as a useful option for the PSA.

\section{Methods}

The cadaver dissection was performed in the Clinical Anatomy Laboratory Nagoya at Nagoya University Graduate School of Medicine. The Ethics Committee of Nagoya University Graduate School of Medicine/Nagoya University Hospital approved the study.

\section{Patient Positioning, Skin Incision, and Muscle Elevations}

The patient is placed supine with an ipsilateral shoulder pad. The head is fixed horizontally with a standard head clamp. A postauricular L-shaped scalp incision is made, beginning at a point $1 \mathrm{~cm}$ posterior to the mastoid tip, passing the asterion, and reaching the temporal squama above the external auditory meatus (EAM) (Fig. 1A). The sternocleidomastoid muscle is elevated with the scalp and retracted anteriorly, and the splenius capitis muscle and other suboccipital muscles are dissected posteroinferiorly to expose the entire mastoid (Fig. 1B). The mastoid emissary vein lies beneath the splenius capitis muscle. In cases in which the vein is large, the vessel wall inside the bony canal is pushed out by packing with an oxidized cellulose

ABBREVIATIONS CPA = cerebellopontine angle; EAM = external auditory meatuS; $L S C=$ lateral semicircular canal; $P S A=$ presigmoid approach; $P S C=$ posterior semicircular canal; $\mathrm{RSA}$ = retrosigmoid approach; $\mathrm{SC}$ = semicircular canal; $\mathrm{SS}$ = sigmoid sinus; $\mathrm{TT}$ = Trautmann's triangle.

SUBMITTED November 24, 2019. ACCEPTED January 20, 2020.

INCLUDE WHEN CITING Published online March 27, 2020; DOI: 10.3171/2020.1.JNS193179. 

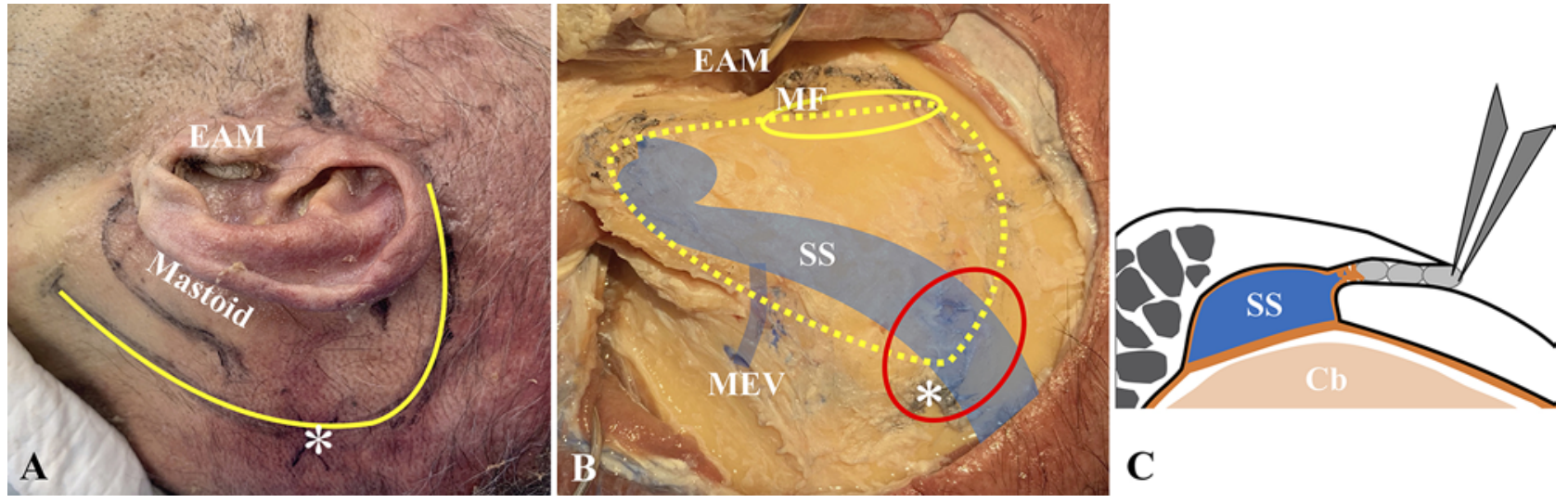

FIG. 1. A: Photograph of a cadaver head. A postauricular L-shaped scalp incision (yellow line) is made beginning at a point $1 \mathrm{~cm}$ posterior to the mastoid tip, passing the asterion and reaching the temporal squama above the EAM. B: Photograph of the entire mastoid after muscle elevation. The area encircled by the red line is drilled away for SS exposure around the asterion. The yellow ellipsoid shows the area drilled out to open the mastoid antrum and expose the dura of the temporal fossa. The yellow dotted line shows the area of cosmetic mastoidectomy. C: Management of the mastoid emissary vein. The vessel wall inside the bony canal is pushed out by packing with an oxidized cellulose ball dipped in fibrin sealant glue. ${ }^{*}=$ asterion; $\mathrm{Cb}=$ cerebellum; MEV = mastoid emissary vein; $\mathrm{MF}=$ mastoid fossa. Figure is available in color online only.

ball dipped in fibrin sealant glue without using bone wax. This maneuver facilitates the safe removal of bone in the later steps (Fig. 1C, not shown in the cadaver dissection).

\section{Mastoidectomy}

A prior lateral suboccipital craniotomy is optional (details not described). The top of the sigmoid sinus (SS) is carefully exposed by drilling around the asterion. The dura of the temporal lobe is also confirmed anterior to the SS. The mastoid antrum is opened by drilling the mastoid fossa, which is almost equal to MacEwen's triangle, and the drilling is directed toward the temporal lobe dura (Fig. 2A). Cosmetic mastoidectomy posterior to the mastoid fossa is made with extreme care regarding the SS after the temporal lobe dura is detached under the temporal squama (Fig. 1B). The SS is carefully detached from the bone that covers it, and the bone is then removed using a ron- geur. This procedure is initiated at the top of the SS and directed downward (Fig. 2B). The SS thus becomes flexible for retraction. TT and the dura of the temporal lobe are then detached from the bone and removal of cancerous bone is continued until the semicircular canals (SCs) are encountered. The bony posterior wall of the EAM is left with a thickness of $5 \mathrm{~mm}$ to avoid injuring the fallopian canal (Fig. 2B and C). Neither exposure of the incus nor dissection of the entire shape of the SCs is necessary. The depth of the fallopian canal is easily identified, equivalent to the line connecting the digastric ridge and lateral semicircular canal (LSC). The endolymphatic sac is coagulated and severed to secure and increase the presigmoid working space. Bone under the fallopian canal is drilled away, taking great care regarding the jugular bulb inferiorly and the posterior semicircular canal (PSC) superiorly (Fig. 3A). The jugular bulb is not exposed unless needed,
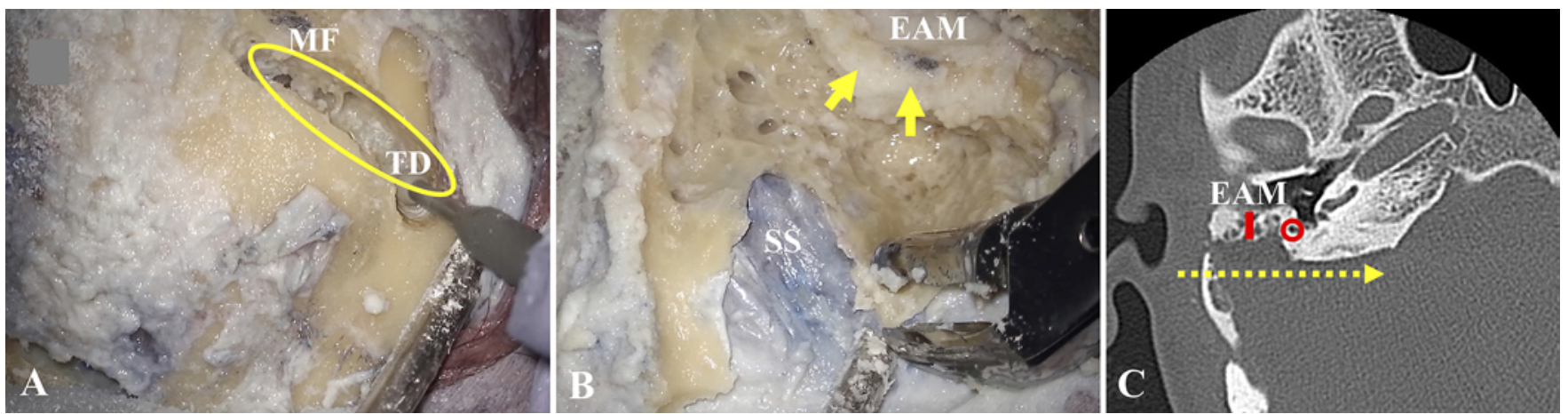

FIG. 2. A and B: Photographs of cadaver dissection. A: The mastoid antrum is opened by drilling the mastoid fossa. Drilling is directed toward the dura of the temporal lobe (yellow ellipsoid, consistent with Fig. 1B). B: The SS is carefully detached from the bone that covers it and the bone is then removed using a rongeur. The procedure is initiated at the top of the SS and directed downward. The bony posterior wall of the EAM is left with a thickness of $5 \mathrm{~mm}$ (yellow arrows). C: CT view of a clinical case showing retention of the bony posterior wall of the EAM. Red circle shows the fallopian canal. Red bar $=5 \mathrm{~mm}$. The remaining 5-mm thickness of the bone does not obstruct the surgical access route (yellow dotted arrow). MF = mastoid fossa; TD = temporal lobe dura. Figure is available in color online only. 

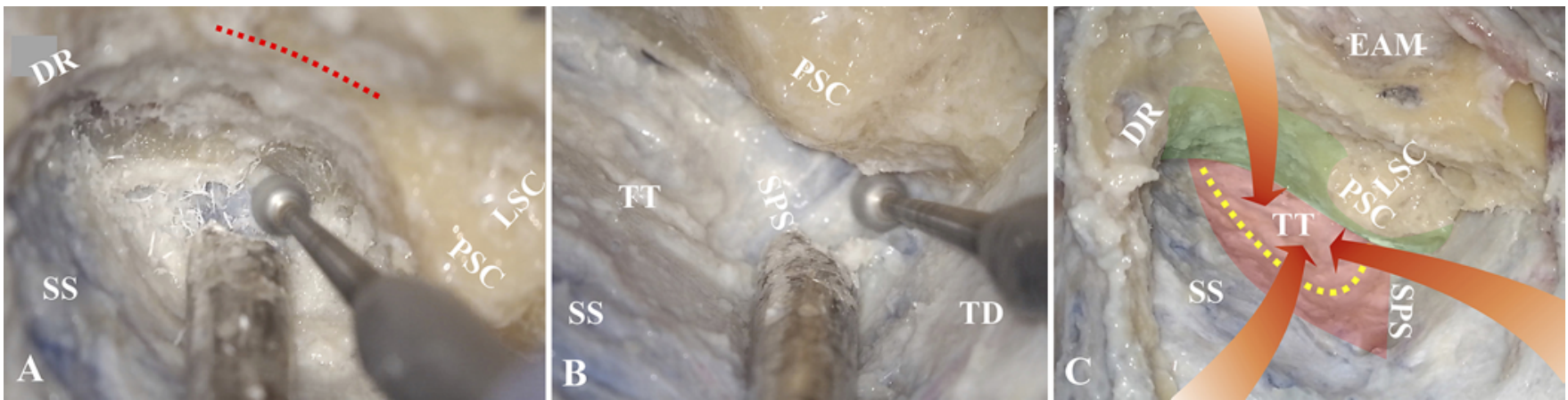

FIG. 3. Photographs of cadaver dissection. A: Bone under the fallopian canal is drilled away. The red dotted line shows the fallopian canal, still covered by surrounding bone. The jugular bulb is not exposed. B: Bone at the sinodural angle is shaved to expose the superior petrosal sinus. C: Photograph showing a view after minimized mastoidectomy. TT (red area) is exposed and the arrows show the approach trajectories to the PSA. A dural incision is made along the SS and superior petrosal vein (yellow dotted line). The SCs are partially exposed and the fallopian canal is not exposed. Further bone removal behind the PSC and fallopian canal (green area) can be performed if deemed necessary for an individual case. DR = digastric ridge; SPS = superior petrosal sinus; $T D=$ temporal lobe dura. Figure is available in color online only.

and bone at the sinodural angle is shaved to expose the superior petrosal sinus (Fig. 3B). Figure 3C shows the view after the minimized mastoidectomy. TT is exposed as a corridor for the PSA. A certain extent of bone removal wider than TT is required to comprehend the orientation inside the mastoid during the procedure and to secure sufficient space for intradural work. Additional bone removal behind the PSC and fallopian canal can be performed if deemed necessary for an individual case.

\section{Dural Incision and Closure}

A dural incision is made along the SS and superior petrosal vein. CSF is evacuated from the CPA cistern. Lateral views of the pons, upper medulla oblongata, and basilar artery are obtained (Fig. 4). The dura is sutured and abdominal fat tissue is packed into the presigmoid space. Lumbar CSF drainage is not necessary for prevention of CSF leakage once a watertight dural closure is achieved. The cortical bone of the mastoid is fixed with titanium plates and the muscles and skin are closed.

The cadaver dissection is shown in Video 1.

VIDEO 1. Cadaveric dissection. Copyright Kuniaki Tanahashi. Published with permission. Click here to view.

\section{Illustrative Cases}

\section{Case 1}

A 66-year-old woman suffered gait disturbance due to a large left CPA epidermoid cyst with intratumoral hemorrhage compressing the brainstem. For safe tumor removal, the standard RSA would be appropriate to reach the tumor extending below the jugular foramen; however, the PSA would be required to observe the tumor where it severely compresses the brainstem and cerebellum. Therefore, the minimized PSA combined with the RSA was selected (Fig. 5A). During presigmoid bone drilling, direct intracranial observation helped us to understand the required extent of bone removal. A surgical view through the PSA complemented the RSA and gross-total resection was achieved (Fig. 5B-D). Symptoms improved markedly, although the patient developed temporary dysphasia after surgery.

\section{Case 2}

A 38-year-old woman developed bilateral abducens nerve palsy and mild left hemiparesis due to a cavernous malformation in the upper medulla oblongata. We consid-

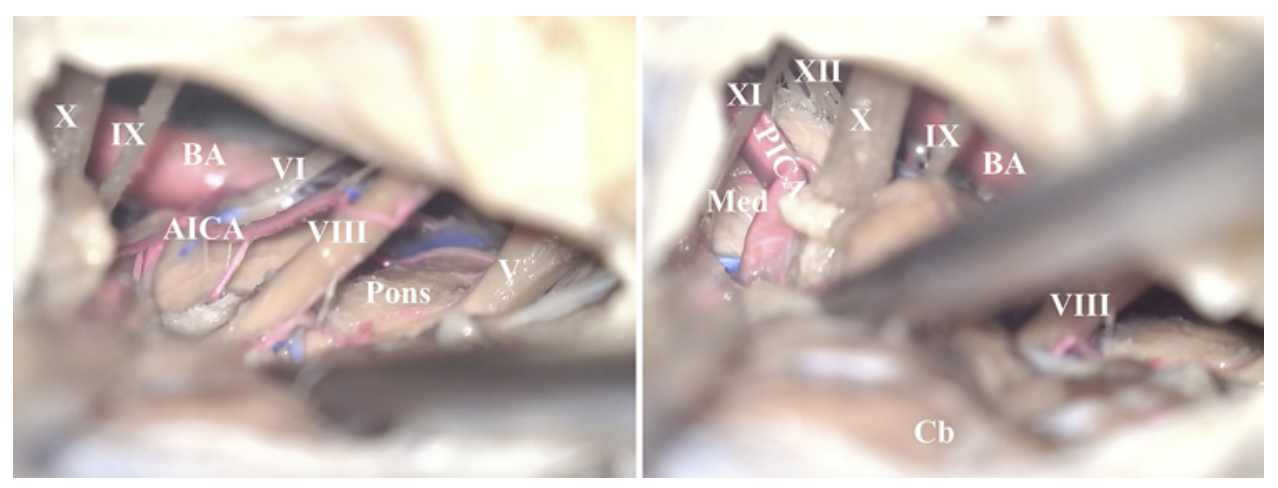

FIG. 4. Photographs of cadaver dissection showing intradural views. The dura is reflected anteriorly. AICA = anterior inferior cerebellar artery; $\mathrm{BA}=$ basilar artery; $\mathrm{Cb}=$ cerebellum; Med = medulla oblongata; $\mathrm{PICA}=$ posterior inferior cerebellar artery; $\mathrm{V}, \mathrm{VI}$, $\mathrm{VIII-XII} \mathrm{=} \mathrm{cranial} \mathrm{nerves} \mathrm{V,} \mathrm{VI,} \mathrm{VIII-XII.} \mathrm{Figure} \mathrm{is} \mathrm{available} \mathrm{in} \mathrm{color} \mathrm{online} \mathrm{only.}$ 

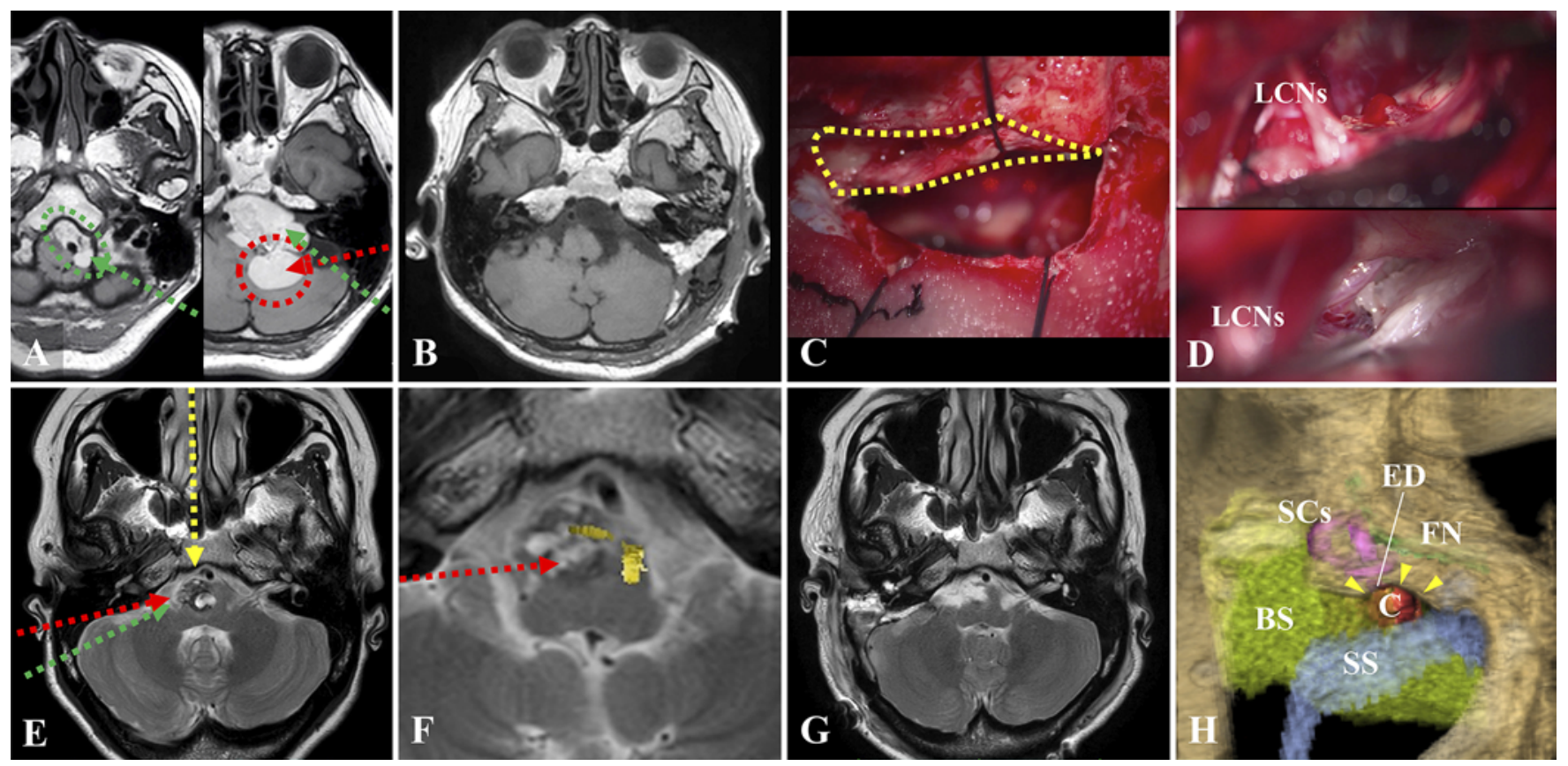

FIG. 5. A-D: Case 1. Results of MRI and operative views for a 66-year-old woman with a large CPA epidermoid cyst containing intratumoral hemorrhage. A: The standard RSA corridor (green dotted arrows) would be appropriate to reach the tumor extending below the jugular foramen (green dotted ellipsoid); however, the PSA trajectory (red dotted arrow) would be required to observe the tumor where it severely compresses the brainstem and cerebellum (red dotted circle). B: Gross-total resection is achieved through the PSA combined with the RSA. C: Bone drilling behind the PSC and fallopian canal (delineated by the yellow dotted line) was performed after an intradural observation. D: A remaining lesion protruding into the brainstem and cerebellum is invisible through the RSA (upper panel), and is directly observed through the PSA (lower panel). E-H: Case 2. Results of MRI and a 3D reconstruction image for a 38 -year-old woman with a cavernous malformation in the upper medulla oblongata. E: The RSA (green dotted arrow), PSA (red dotted arrow), or endonasal transclival approach (yellow dotted arrow) could be chosen for the surgical corridor. F: Diffusion tensor imaging tractography indicates that the PSA (red dotted arrow) could provide a sufficient view of the deeply seated lesion to avoid injuring the ventrally located pyramidal tract. G: Endoscopic resection is performed via the PSA, achieving gross-total resection. $\mathrm{H}$ : A 3D reconstruction image of preoperative MRI and postoperative CT demonstrates minimal bone removal for access to the cavernous malformation (yellow arrowheads). $B S=$ brainstem; $C=$ cavernous malformation; $E D=$ endolymphatic duct; FN = facial nerve; LCNs = lower cranial nerves. Figure is available in color online only.

ered that lateral access through the PSA would be the optimal trajectory to obtain an adequate view of the deeply seated lesion and to avoid injuring the pyramidal tract, which was located in the ventral medulla (Fig. 5E and F). Endoscopic resection was performed via the minimized PSA. To obtain a straight corridor to the lesion, bone removal behind the PSC and fallopian canal under the assistance of neuronavigation was performed in the last stage of the approach. Gross-total resection was achieved and only mild right abducens nerve palsy remains as of 6 months after surgery (Fig. 5G and H). The surgery is depicted in Video 2.

VIDEO 2. Case 2. Endoscopic resection performed via the minimized PSA in a woman with a cavernous malformation. Copyright Kuniaki Tanahashi. Published with permission. Click here to view.

\section{Discussion}

The PSA is applicable to large CPA tumors compressing the brainstem, anterolateral pontine cavernous malformations, and vertebrobasilar artery aneurysms..$^{1-4}$ In contrast to its effectiveness, the approach can be considered time-consuming and is accompanied by a higher risk of complications compared to the standard RSA. We estab- lished a method for simplified mastoidectomy in the PSA, minimizing bone removal with a focus on exposing TT, the presigmoid dura mater bordered by the superior petrosal sinus, SS, jugular bulb, and PSC, ${ }^{6}$ as an access route for the PSA. Unnecessary destruction of crucial structures can be avoided by limiting mastoidectomy (Fig. 3C).

To reduce operating time, it is essential to detach the SS from the bone covering and then remove the bone beginning at the top of the SS and moving downward (Fig. 2B). ${ }^{7}$ The risk of SS injury during eggshell drilling can be spared and the presigmoid working space is widened by SS moblization. ${ }^{8}$ Furthermore, transection of the endolymphatic sac, which appears to be a dural fold behind the PSC, makes the presigmoid space wider. An experimental study demonstrated that no long-term loss of cochlear function developed after endolymphatic sac ablation. ${ }^{9}$ Preservation of the bone covering the posterior wall of the EAM is also important. Location of the vertical portion of the facial nerve is reported to be within $5 \mathrm{~mm}$ of the posterior wall of the EAM (Fig. $2 \mathrm{~B}$ and C).$^{10}$ Preservation of the bone not only reduces the operating time, but also decreases the risk of facial nerve injury. In addition, full exposure of the SCs is unnecessary. In case 2, the SCs 
were covered by thick cortical bone and only the LSC was slightly exposed (Video 2). Management of the mastoid emissary vein also helps avoid bleeding and accelerates removal of bone around the vein by pushing the vessel wall out of the bony canal (Fig. 1C).

To preserve the SCs and facial nerve, it is helpful to confirm anatomical landmarks such as the LSC, digastric ridge, endolymphatic sac, and thickness of the bony posterior wall of the EAM in order to predict the location of the PSC and fallopian canal, ${ }^{2,6,10,11}$ which could be close to the drilling point in the last step of mastoidectomy. Preoperative assessment of pneumatization around the SCs and the thickness of the bone behind the PSC on CT is also essential given their variety. ${ }^{8}$ Even though neuronavigation can enhance the understanding of the orientation and minimize bone removal (Fig. 5H), overuse should be avoided due to its potential for error. ${ }^{12}$

Regarding operative views through the PSA, lateral access to the CPA, pons, upper medulla, and basilar artery is obtained (Fig. 4). Minimal mastoidectomy appears sufficient to secure access to the safe entry zones for pons and upper medulla cavernous malformations, ${ }^{13}$ although the extent of bone removal can be tailored to an individual case (Fig. 3C). As seen in the operative cases, bone removal behind the PSC and fallopian canal could be conducted with the assistance of direct intradural observation in case 1 and neuronavigation in case 2 (Fig. 5). In cases in which the lesion shows supratentorial extension or lower extension below the jugular foramen, the use of another approach or a combination with another approach should be considered. ${ }^{1,12,14}$

The PSA should be performed in collaboration with neuro-otologists who are familiar with the temporal bone, and it can be considered as one of the options when deciding a surgical strategy. However, it is recommended that a neurosurgeon perform this technique because he or she is familiar with intracranial work. Mastoidectomy can only be minimized with a suitable understanding of the required surgical corridor and available working space.

\section{Conclusions}

Our TT-focused mastoidectomy could maximize the simplicity and safety of mastoidectomy, reduce operating time, and provide a sufficient operative view for the PSA.

\section{References}

1. Day JD, Fukushima T, Giannotta SL. Cranial base approaches to posterior circulation aneurysms. J Neurosurg. 1997;87(4):544-554.

2. Graffeo CS, Peris-Celda M, Perry A, et al. Anatomical stepby-step dissection of complex skull base approaches for trainees: surgical anatomy of the posterior petrosal approach. J Neurol Surg B Skull Base. 2019;80(4):338-351.

3. Hauck EF, Barnett SL, White JA, Samson D. The presigmoid approach to anterolateral pontine cavernomas. Clinical article. J Neurosurg. 2010;113(4):701-708.
4. Iacoangeli M, Salvinelli F, Di Rienzo A, et al. Microsurgical endoscopy-assisted presigmoid retrolabyrinthine approach as a minimally invasive surgical option for the treatment of medium to large vestibular schwannomas. Acta Neurochir (Wien). 2013;155(4):663-670.

5. Wong AK, Stamates MM, Bhansali AP, et al. Radiographic assessment of the presigmoid retrolabyrinthine approach. Surg Neurol Int. 2017;8:129.

6. Tubbs RS, Griessenauer C, Loukas M, et al. Trautmann's triangle anatomy with application to posterior transpetrosal and other related skull base procedures. Clin Anat. 2014;27(7):994-998.

7. Goto T, Ishibashi K, Morisako H, et al. Simple and safe exposure of the sigmoid sinus with presigmoid approaches. Neurosurg Rev. 2013;36(3):477-482.

8. Alonso F, Dekker SE, Wright J, et al. The retrolabyrinthine presigmoid approach to the anterior cerebellopontine region: expanding the limits of Trautmann triangle. World Neurosurg. 2017;104:180-185.

9. Swart JG, Schuknecht HF. Long-term effects of destruction of the endolymphatic sac in a primate species. Laryngoscope. 1988;98(11):1183-1189.

10. Zaghal ZA, Raad RA, Nassar J, et al. Anatomic relationship between the facial nerve and the tympanic annulus. Otol Neurotol. 2014;35(4):667-671.

11. Wanibuchi M, Friedman AH, Fukushima T. Photo Atlas of Skull Base Dissection. New York: Thieme Medical Publishers; 2008:272-276.

12. Cinibulak Z, Krauss JK, Nakamura M. Navigated minimally invasive presigmoidal suprabulbar infralabyrinthine approach to the jugular foramen without rerouting of the facial nerve. Neurosurgery. 2013;73(1)(Suppl Operative):ons3ons15.

13. Cavalcanti DD, Preul MC, Kalani MY, Spetzler RF. Microsurgical anatomy of safe entry zones to the brainstem. J Neurosurg. 2016;124(5):1359-1376.

14. Behari S, Tyagi I, Banerji D, et al. Postauricular, transpetrous, presigmoid approach for extensive skull base tumors in the petroclival region: the successes and the travails. Acta Neurochir (Wien). 2010;152(10):1633-1645.

\section{Disclosures}

Dr. Uda received clinical or research support for the study described (includes equipment or material) from Mizuho Corp.

\section{Author Contributions}

Conception and design: Tanahashi. Acquisition of data:

Tanahashi, Uda, Araki, Takeuchi, Choo, Chalise, Motomura,

Ohka. Analysis and interpretation of data: Tanahashi, Uda, Araki. Drafting the article: Tanahashi. Critically revising the article: Natsume. Reviewed submitted version of manuscript: all authors. Study supervision: Wakabayashi, Natsume.

\section{Supplemental Information \\ Videos}

Video 1. https://vimeo.com/389021236.

Video 2. https://vimeo.com/389021256.

\section{Correspondence}

Kuniaki Tanahashi: Nagoya University Graduate School of Medicine, Nagoya, Japan.tanahk@med.nagoya-u.ac.jp. 